\title{
Open wedge high tibial osteotomy for the treatment of osteoarthritis of both knee and ankle in the same leg: A report of two cases
}

\author{
Ji-Hoon Baek ${ }^{1}$, Su Chan Lee ${ }^{1}$, Hosun Jin ${ }^{1}$, Hye Sun Ahn ${ }^{1}$, and Chang Hyun Nam ${ }^{1}$ \\ ${ }^{1}$ Joint \& Arthritis Research, Himchan Hospital
}

February 3, 2021

\begin{abstract}
We report two cases of patients with concurrent osteoarthritis of the knee and the ipsilateral ankle. Patients were treated with high tibial osteotomy (HTO). In well-planned and selected cases, HTO may be effective in treating not only the knee but also the ankle in patients with ipsilateral double-joint osteoarthritis.
\end{abstract}

\section{INTRODUCTION}

High tibial osteotomy (HTO) has been an effective surgical method for the treatment of moderate osteoarthritis of the medial compartment of the knee. ${ }^{1,2,3}$ Re-alignment of a varus deformity of the knee by HTO or total knee arthroplasty (TKA) inevitably changes the alignment of the ankle joint. ${ }^{4,5}$ However, the effects of these procedures on the ankle joint have not been well described.

Patients who suffer from degenerative osteoarthritis in both knee and ankle joints on the same limb are occasionally encountered in clinical practice. Osteoarthritis of the ankle is relatively rare compared to that of the knee. ${ }^{6}$ Moreover, patients with concurrent osteoarthritis and a varus deformity of both the knee and ankle in the same leg are extremely rare. ${ }^{2}$ Here, we reported two cases of patients with concurrent osteoarthritis of the knee and the ipsilateral ankle in whom an HTO was performed, resulting in significant clinical and radiographic improvement.

\section{CASE REPORT}

\subsection{Case 1}

A 48-year-old female patient who complained of serious pain in the right knee and ipsilateral ankle for about three years presented to our clinic in January 2019. She had no systemic musculoskeletal disease or other medical history. She was $150.0 \mathrm{~cm}$ in height and $57.0 \mathrm{~kg}$ in weight, with a body mass index of 25.3 $\mathrm{kg} / \mathrm{m}^{2}$. Pain in both the knee and ankle joints began three years ago due to a moderate varus deformity and osteoarthritis of the double joints. Physical examination showed that the right knee could actively flex to $120^{\circ}$ and had a prominent hyperextension of about $5^{\circ}$. The Hospital for Special Surgery (HSS) score of the right knee was 65. In addition, the right ankle was swollen and showed abnormal plantar flexion and was markedly varus. The osteoarthritic ankle stage was Takakura's classification stage $3 .^{7}$ Hip and pelvis movements were symmetrical, full, and pain-free. The results of the spine examination were normal with no neurological abnormalities.

Kellgren-Lawrence (K-L) grade II ${ }^{\sim}$ III osteoarthritic change of the right knee was documented. The weightbearing X-ray showed the varus deformity, and the hip-knee-ankle (HKA) angle was $8.2^{\circ}$. Her ankle was noted to have $16.4^{\circ}$ of varus talar tilt (TT) and $6.1^{\circ}$ of tibial inclination (TI) (Fig. 1). 
We considered that her ankle symptoms could be attributed to the varus alignment of the left knee. Through a medial approach above the pes anserinus, an opening wedge HTO was performed. Intraoperatively, the medial osteotomy wedge was opened to $9^{\circ}$ and the desired correction was achieved. The patient recovered without any complications and was advised to perform non-weight-bearing mobilization with a walker during rehabilitation.

The patient was satisfied with the outcome of her surgery, reporting a marked reduction in pain and improved stability of both knee and ankle joints six weeks postoperatively. Three months later, there was no pain in her left knee, and she could walk unaided. Her active range of motion was 0 to $135^{\circ}$. The HSS score of the right knee was 85 three months after surgery and she volunteered a subjective improvement in ankle pain of $95 \%$.

The postoperative weight-bearing X-ray showed favorable limb alignment on the coronal view. The standing HKA angle improved from a preoperative value of $8.2^{\circ}$ to $0^{\circ}$ at follow-up. The TT was corrected from $16.4^{\circ}$ to $8.2^{\circ}$ and the TI from $6.1^{\circ}$ to $0.7^{\circ}$ (Fig. 2).

\subsection{Case 2}

A 59-year-old female patient who complained of serious pain in the left knee and ipsilateral ankle for about one year presented at our clinic in July 2019. She had no systemic musculoskeletal disease or other medical history. She was $157.0 \mathrm{~cm}$ in height and $62.0 \mathrm{~kg}$ in weight, with a body mass index of $25.2 \mathrm{~kg} / \mathrm{m}^{2}$. The HSS score of the left knee was 69 . The osteoarthritic ankle stage was Takakura's classification stage 3.

Kellgren-Lawrence (K-L) grade II ${ }^{\sim}$ III osteoarthritic change of the left knee was documented. The weightbearing X-ray showed the varus deformity, and the hip-knee-ankle (HKA) angle was $4.5^{\circ}$. Her ankle was noted to have $19.5^{\circ}$ of varus TT and $5.2^{\circ}$ of TI (Fig. 3).

Through a medial approach above the pes anserinus, an opening wedge HTO was performed. Intraoperatively, the medial osteotomy wedge was opened to $6^{\circ}$ and the desired correction was achieved. The pain on the knee and ankle joints had disappeared three months after HTO. The HSS score of the left knee was 85 three months after surgery.

The postoperative weight-bearing X-ray showed favorable limb alignment on the coronal view. The standing HKA angle improved from a preoperative value of $4.5^{\circ}$ to $-1.4^{\circ}$ at follow-up. The TT was corrected from $19.5^{\circ}$ to $10.6^{\circ}$ and the TI from $5.2^{\circ}$ to $0.5^{\circ}$ (Fig. 4 ).

\section{DISCUSSION}

In these cases, HTO was an effective procedure to treat medial osteoarthritis in co-directional varus deformities at the knee and ankle of the same leg. Re-alignment of the proximal tibia leads to changes in ankle pressure, which could be beneficial in guiding the treatment of patients with medial osteoarthritis of the ankle.

HTO may influence the alignment and symptoms of the ankle joint. The effects of the HTO on the biomechanics of the ankle joint remain controversial. Several studies reported that the correction of severe varus deformity of the knee joint with HTO or TKA could result in pain and osteoarthritis progression of the ankle joint. ${ }^{5,8,9}$ Jeong and SooHoo ${ }^{8}$ reported the case of a patient with persistent ankle pain after bilateral HTO for the treatment of osteoarthritis in both knee joints, which was treated with a corrective supramalleolar osteotomy of the ankle. Lee and Jeong ${ }^{5}$ reported that $21.8 \%$ of the patients had newly developed or progressive ankle arthritis after TKA in a series of 142 TKA cases. Graef et al. ${ }^{9}$ reported that the excessive correction of knee malalignment after TKA was associated with worse postoperative function in the ankle joint. In contrast, Takeuchi et al. ${ }^{10}$ reported the successful treatment of 16 HTOs in ten patients with osteoarthritis of the ipsilateral knee and ankle joints. Elson et al. ${ }^{11}$ presented the case of a patient with medial ankle pain and lateral instability in whom an HTO was performed, resulting in significant resolution of the ankle symptoms. Recently, Kim et al. ${ }^{12}$ reported that the correction of a knee varus deformity with HTO or TKA could improve the symptoms of medial ankle arthritis but could worsen lateral ankle arthritis. These 
reports corresponded to improvements in the symptoms of medial osteoarthritis in the ankle joint following HTO in our cases.

In well-planned and selected cases, HTO may be effective in treating not only the knee but also overload of the ankle in patients with ipsilateral double-joint osteoarthritis.

\section{ACKNOWLEDGMENTS}

This work was supported by our institution. Published with written consent of the patient.

\section{CONFLICT OF INTEREST}

Each author certifies that he or she has no commercial association (eg. consultancies, stock ownership, equity interest, patent, licensing arrangements, etc.) that might pose a conflict of interest in connection with the submitted article.

\section{AUTHOR CONTRIBUTIONS}

Ji-Hoon Baek and Chang Hyun Nam: writing and revision of article. Su Chan Lee, Hosun Jin and Hye Sun Ahn: data collection and statistical analysis.

\section{ETHICAL APPROVAL}

This study was approved by the Institutional Review Board of Himchan hospital.

\section{DATA AVAILABILITY STATEMENT}

The data that support the findings of this study are available from the corresponding author upon reasonable request.

\section{REFERENCES}

1. Koshino T. Clinical findings and etiology of osteoarthritis of the knee. Asian Med J . 1987;30:623-629.

2. Matthews LS, Goldstein SA, Malvitz TA, Katz BP, Kaufer H. Proximal tibial osteotomy. Factors that influence the duration of satisfactory function. Clin Orthop Relat Res . 1988;229:193-200.

3. Moreland JR, Bassett LW, Hanker GJ. Radiographic analysis of the axial alignment of the lower extremity. J Bone Joint Surg Am . 1987;69(5):745-749.

4. Chandler JT, Moskal JT. Evaluation of knee and hindfoot alignment before and after total knee arthroplasty: a prospective analysis. J Arthroplasty . 2004;19(2):211-216.

5. Lee JH, Jeong BO. Radiologic changes of ankle joint after total knee arthroplasty. Foot Ankle Int . 2012;33(12):1087-1092.

6. Tanaka Y, Takakura Y, Hayashi K, Taniguchi A, Kumai T, Sugimoto K. Low tibial osteotomy for varustype osteoarthritis of the ankle. J Bone Joint Surg Br . 2006;88(7):909-913.

7. Takakura Y, Tanaka Y, Sugimoto K, Tamai S, Masuhara K. Ankle arthroplasty. A comparative study of cemented metal and uncemented ceramic prostheses. Clin Orthop Relat Res . 1990;252:209-216.

8. Jeong BO, SooHoo NF. Ankle Deformity After High Tibial Osteotomy for Correction of Varus Knee: A Case Report. Foot Ankle Int . 2014;35:725-729.

9. Graef F, Falk R, Tsitsilonis S, Perka C, Zahn RK, Hommel H. Correction of excessive intraarticular varus deformities in total knee arthroplasty is associated with deteriorated postoperative ankle function. Knee Surg Sports Traumatol Arthrosc . 2020;28:3758-3765.

10. Takeuchi R, Saito T, Koshino T. Clinical results of a valgus high tibial osteotomy for the treatment of osteoarthritis of the knee and the ipsilateral ankle. Knee. 2008;15(3):196-200. 
11. Elson DW, Paweleck JE, Shields DW, Dawson MJ, Ferrier GM. Stretching the indications: high tibial osteotomy used successfully to treat isolated ankle symptoms. BMJ Case Rep . 2013;2013:bcr2013200527.

12. Kim JG, Suh DH, Choi GW, Koo BM, Kim SG. Change in the weight-bearing line ratio of the ankle joint and ankle joint line orientation after knee arthroplasty and high tibial osteotomy in patients with genu varum deformity. Int Orthop . 2021;45(1):117-124.

\section{Figure Legends}

FIGURE 1. Before HTO. Whole leg radiographs of a 48-year-old woman in the standing position. a . Hipknee-ankle (HKA) angle: the angle between the femoral and tibial mechanical axes in the anteroposterior view. $\mathbf{b}$. Talar tilt (TT): the angle between the tangent of the superior talar surface and the horizontal line. Tibial inclination (TI): the angle between the tibial axis and the perpendicular line.

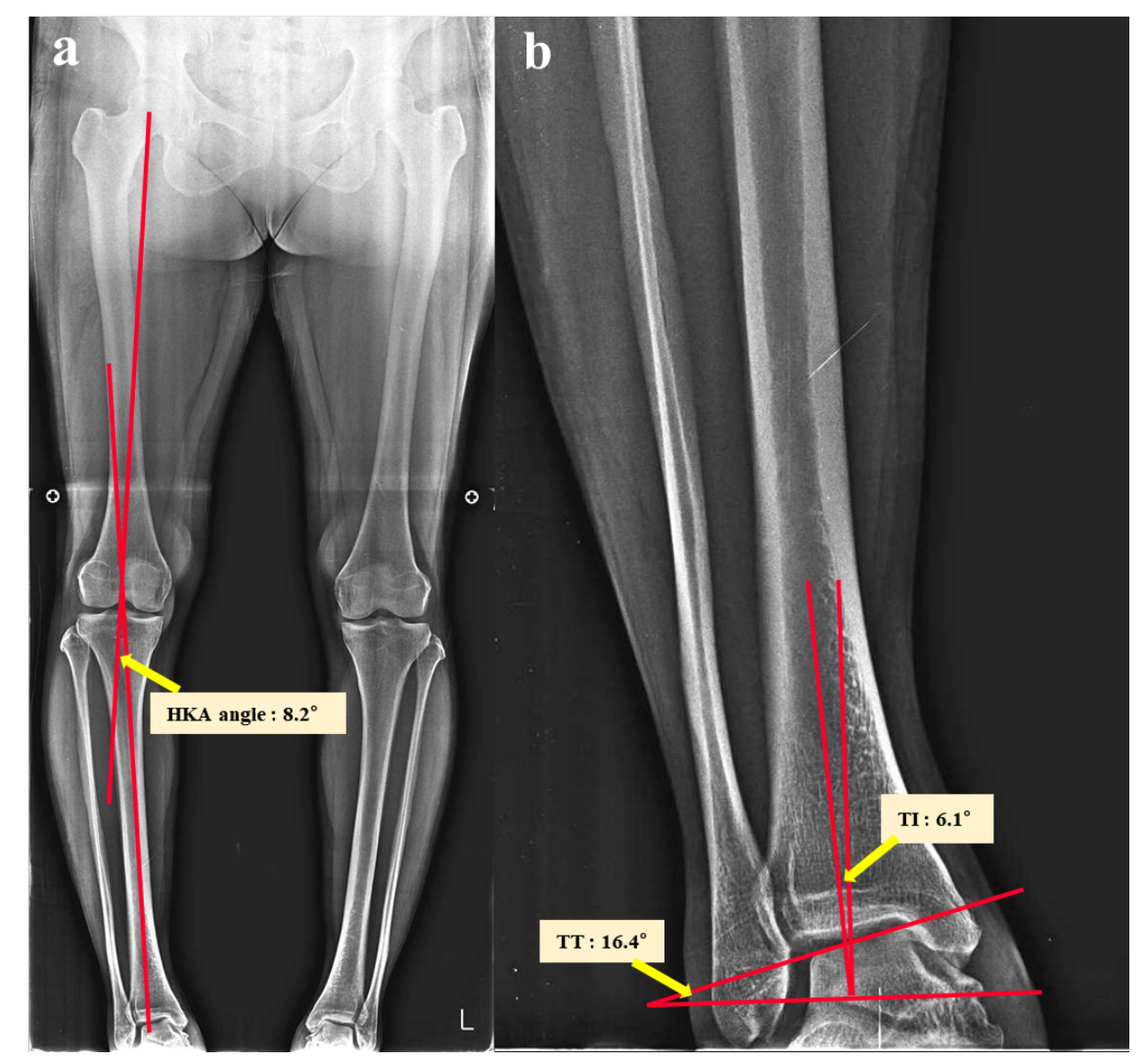

FIGURE 2. One year after HTO. Whole leg radiographs of a 48-year-old woman in the standing position. a . HKA angle. b. TT and TI. 


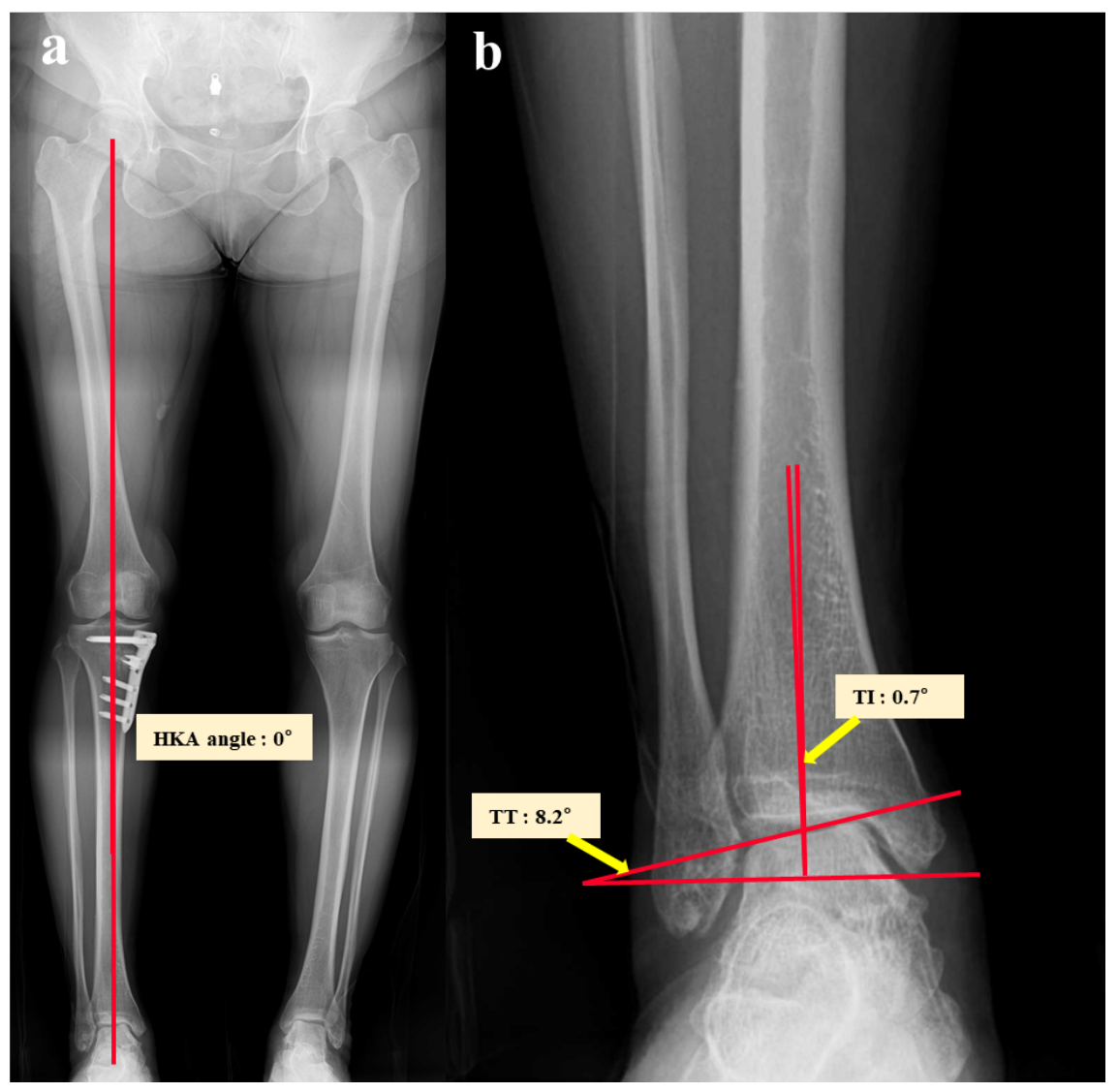

FIGURE 3. Before HTO. Whole leg radiographs of a 59-year-old woman in the standing position. a . HKA angle. $\mathbf{b}$. TT and TI. 


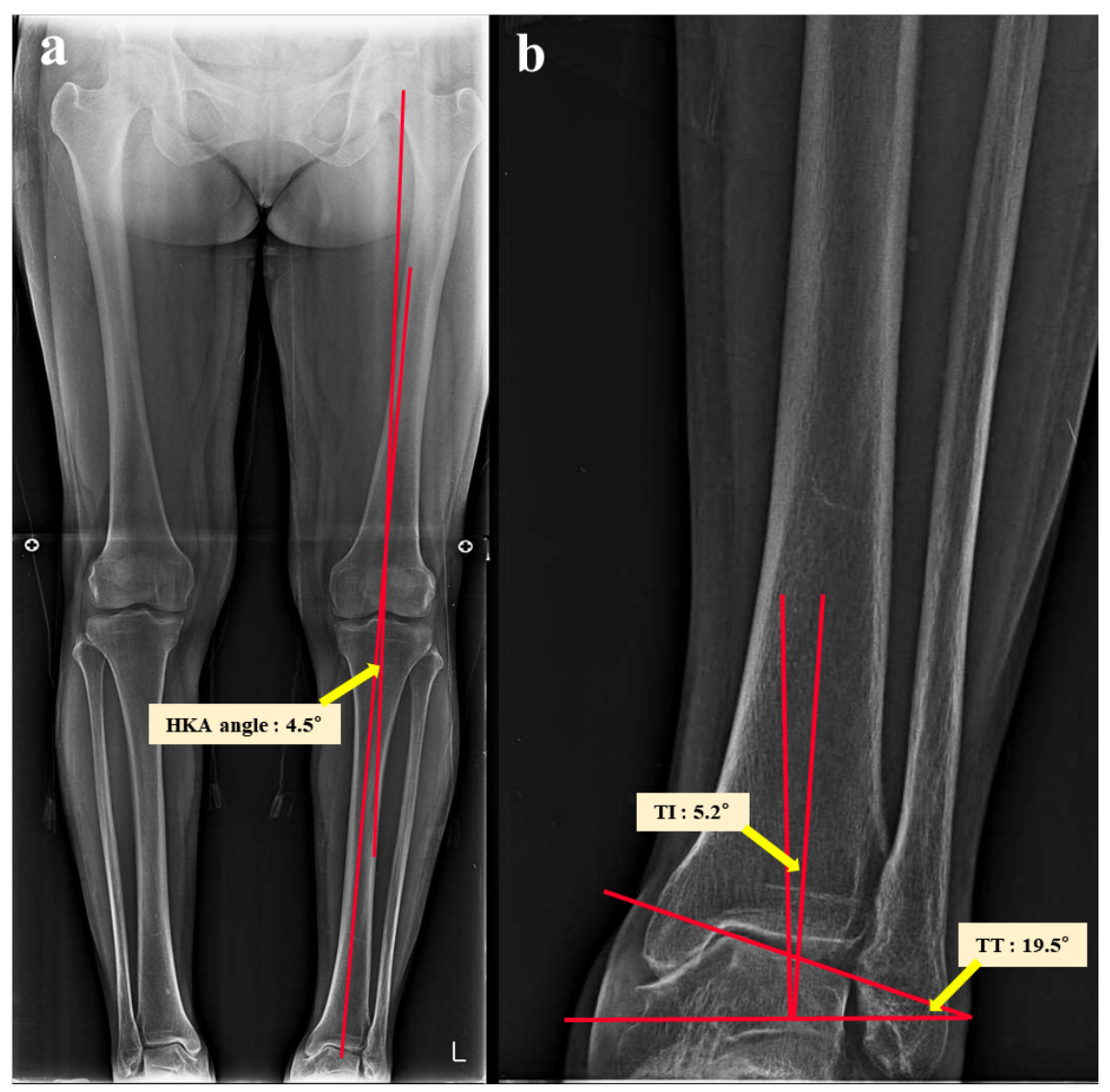

FIGURE 4. One year after HTO. Whole leg radiographs of a 59-year-old woman in the standing position. a . HKA angle. b. TT and TI. 


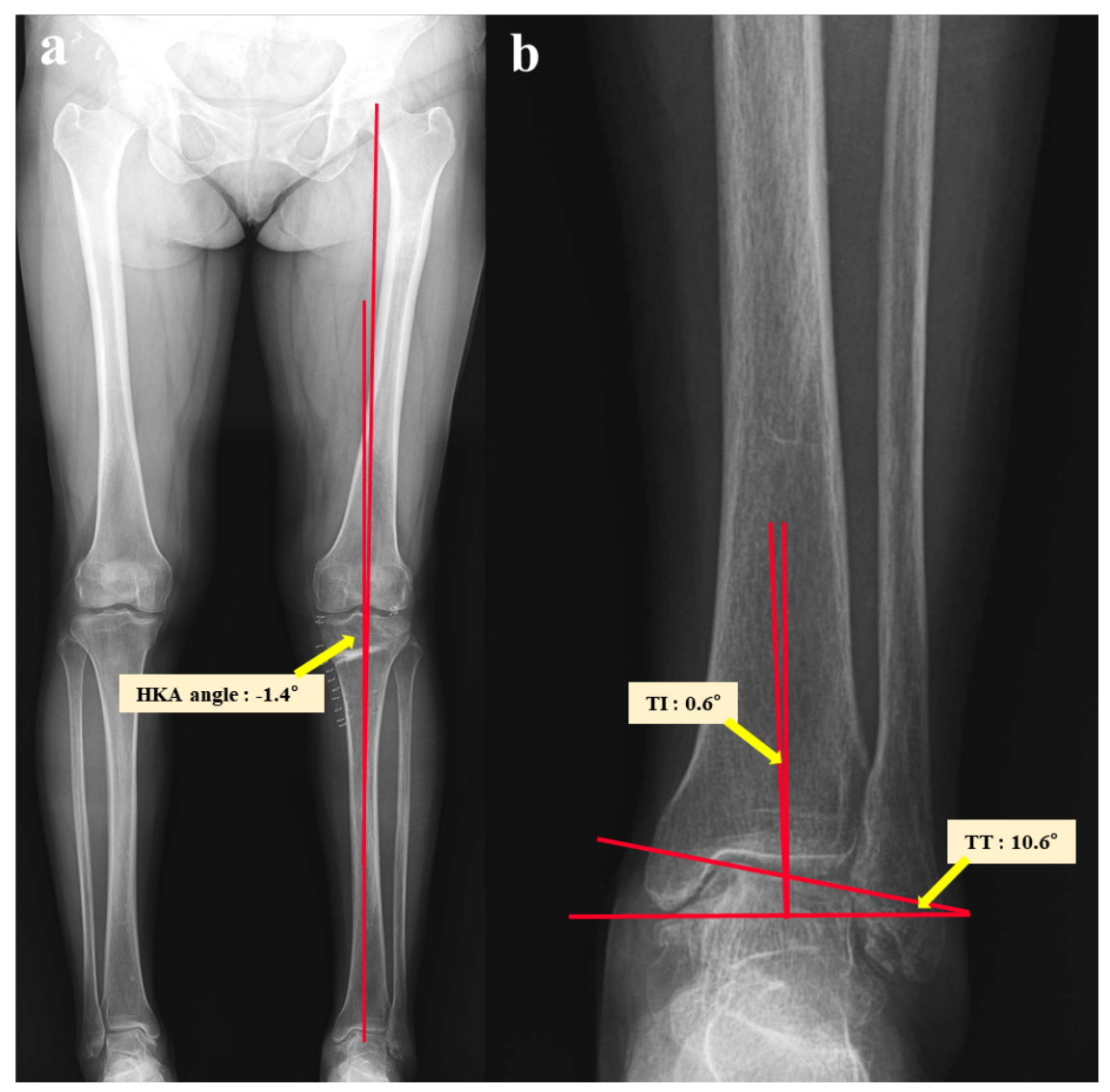

